\title{
EL EXCESO COMERCIAL COMO CAUSA DE LA CRISIS DEL MUNDO GLOBAL EN LAS DEPRESIONES DE 1929 Y 2008. LA SITUACIÓN $D E$ ESPAÑA
}

\section{COMMERCIAL EXCESS AS A CAUSE OF THE GLOBAL WORLD CRISIS IN THE DEPRESSIONS OF 1929 AND 2008. THE SITUATION IN SPAIN}

\author{
Javier Aranda Prieto*
}

RESUMEN

Las crisis de los años 1929 y 2008, aunque alejadas en el tiempo, coinciden en que ambas pusieron en duda el mundo económico global. En el marco temporal de la crisis económica del 2008, el objetivo de este artículo es entender que esta depresión global se origina en el exceso del volumen comercial provocado por una aceleración continua de la producción. Esto causó un exceso de producción acumulado en los almacenes de las empresas, lo cual indujo a la inestabilidad de la red empresarial en las diferentes naciones como sucedió en países como España. En el caos social causado por el exceso, las empresas apostaron por una serie de políticas conservadoras como el recorte salarial y los despidos masivos que provocaron el endeudamiento crónico de las economías domésticas. Como conclusión, el paulatino crecimiento de la oferta comercial ha configurado un mundo financiero interconectado a través de la desigualdad social desde el empobrecimiento del negocio local en beneficio de las multinacionales. Esto ha fracturado la sociedad global, y por consiguiente, aumentado la incertidumbre de un mundo globalizado muy inestable.

PALABRAS CLAVE: COMERCIO * PRODUCCIÓN * CAPITALISMO * TECNOLOGÍA * EMPRESA

\section{ABSTRACT}

The crises of the years 1929 and 2008, although distant in time, coincide in that they both cast doubt on the global economic world. By analyzing the time frame of the 2008 economic crisis, the objective of this article is to understand that the origin of this global depression was caused by excess trade volume from a continuous acceleration of production. This caused accumulated excess production in warehouses of companies, and with it, induced the instability of the business network in different nations, as happened 
in countries like Spain. In the social chaos caused by excess, companies opted for a series of conservative policies such as the wage cut and the massive layoffs that caused the chronic indebtedness of the domestic economy. As a conclusion, the gradual growth of the commercial offer has shaped an interconnected financial world through social inequality since the impoverishment of the local business for the benefit of the multinationals, thereby fracturing the global society, and therefore increasing the uncertainty of a very unstable globalized world.

KEYWORDS: COMMERCE * PRODUCTION * CAPITALISM * TECHNOLOGY * COMPANY

\section{UNA BREVE EXPLICACIÓN DE LAS CRISIS ECONÓMICAS DE 1929 Y 2008}

El mundo globalizado se ha visto comprometido económica y socialmente después de la crisis de 2008, tal y como sucedió en una primera etapa globalizadora al originarse la crisis del año 1929, la cual inició la Gran Depresión de los años 30 del siglo pasado. En estas dos etapas coincide el desmesurado crecimiento del comercio de mercancías, lo cual provoca un exceso de producción acumulado que desencadena, por un lado, un descenso de solidez de la red empresarial de los diferentes países, y por otro lado, constituye que se realicen políticas de recortes salariales y sucesivos despidos que repercuten en un endeudamiento crónico que afecta la economía doméstica de las naciones. En este contexto, España no ha sido ajena a esta situación.

El problema del capitalismo del siglo xx y principios del xxI, según Carlos Marichal (2010), ha sido encontrar el equilibrio entre una producción acelerada mediante las diferentes revoluciones industriales a partir del desarrollo tecnológico y las mejoras salariales necesarias para absorber toda la oferta creada con el objetivo de crear más demanda. Este desequilibrio establece un creciente endeudamiento que actúa como un generador crónico de continuas crisis económicas de menor o mayor grado. Las dos crisis han afectado en mayor medida en la historia del mundo contemporáneo han sido las de 1929 y la más reciente del 2008, las cuales se desarrollan a continuación (Marichal, 2010).

El crac de 1929 fue el momento socioeconómico más importante del siglo xx. Giovanni Arrighi y Beverly Silver (2001) explican como la caída de Gran Bretaña como centro hegemónico del mundo, después de la crisis de 1873, acumuló excedentes productivos por encima de lo que podía invertirse rentablemente en la expansión del comercio y la producción. Esta situación se agravó con los costes astronómicos de la Primera Guerra Mundial que destruyeron las finanzas británicas. Estados Unidos, como señala Dominique Barjot (2005), se convirtió entonces en la primera potencia mundial debido a que, en los años 80 del siglo xix, una red de transportes eficientes permitió emerger un mercado de consumo de masas debido a la penuria de mano de obra que obligó a ofrecer salarios elevados, más que Gran Bretaña, Francia y Alemania. Esto provocó un aumento del consumo en los productos no alimenticios que coincidió con el auge de la industria del automóvil. La industria norteamericana favoreció un modelo de crecimiento orientado a incrementar el nivel de la productividad del trabajo. Una corriente masiva de inversiones favoreció la aparición de la gran empresa multidivisional $y$ de gestión. La organización funcional, con un mayor grado de especialización en la producción, fue orientada hacia la clientela y organizada según una lógica de línea de productos. Sus efectos fueron visibles en las economías de escala, las economías de diversificación y las economías de transacción (Barjot, 2005).

De acuerdo con Barjot (2005), la elevada productividad provocó una sensible reducción de los costos, lo cual permitió una rápida expansión del mercado interior. Todo ello estuvo sustentado en un progreso técnico perceptible en términos de innovación siendo muy favorables para los Estados Unidos. La Primera Guerra Mundial reforzó la posición 
económica internacional de los Estados Unidos respecto a las naciones europeas como el Reino Unido y, sobre todo, Alemania, Francia y Rusia. El modelo norteamericano se convirtió en la referencia, tanto en materia económica como financiera, debido al endeudamiento de los países occidentales por la Gran Guerra. Los norteamericanos buscaron una solución al problema financiero por las reparaciones en Europa después de la Primera Guerra Mundial, planeando y concediendo 2 planes económicos como fueron el Plan Dawes (1924) y el Plan Young (1929), con el objetivo de reestablecer el estatus financiero europeo anterior a la guerra. La finalidad era provocar el aumento de la demanda comercial, pero la crisis de los años 30 detuvo la iniciativa y el mundo globalizado desapareció, en parte porque los países europeos y sus instituciones no supieron manejar las consecuencias psicológicas de un mundo interconectado (Harold, 2003).

La crisis económica mundial del 2008 sucedió 69 años después de la Gran Depresión de los años 30 del siglo pasado. Esta crisis tuvo origen de nuevo en los Estados Unidos como centro hegemónico y los principales factores que se atribuyen como causas del trance financiero se encuentran: los fallos en la regulación económica en los países afectados, la gran cantidad de delitos cometidos por los bancos, la sobrevalorización de productos, la crisis alimentaria mundial y energética, así como, una crisis crediticia-hipotecaria y de confianza en los mercados (Tapia y Astarita, 2011).

Los primeros síntomas aparecieron un año antes, en agosto de 2007, con la quiebra de varios bancos menores de inversión agravándose en el 2008, tras la caída del banco estadounidense Lehman Brothers, desplome debido, en parte, a la crisis de las hipotecas subprime, que representaban un alto porcentaje de la inversión. Por esta situación, las bolsas y los mercados de valores se derrumbaron provocando la crisis financiera mundial de 2008, tanto por la crisis norteamericana como por el hecho de estar dentro de un contexto económico global. Posteriormente, los distintos gobiernos del mundo tuvieron que realizar numerosos rescates financieros para salvar a empresas financieras y no financieras de una probable quiebra en sus respectivos países, lo cual convirtió la deuda, especialmente en los países de la eurozona, en una de las consecuencias más graves de la crisis económica, ya que debido a la gran cantidad de dinero asignado a los rescates financieros $y$ a la fuerte caída generalizada de los ingresos en la recaudación fiscal, algunos gobiernos se vieron obligados a realizar programas de austeridad económica que implicaron fuertes recortes sociales que provocaron contestaciones sociales por el aumento de la desigualdad $y$ la pobreza generalizada (Loayza, 2012).

\section{EL EXCESO DE PRODUCCIÓN EN 1929 DESDE UNA VISIÓN PERIFÉRICA-LOCAL. EL CASO DE ESPAÑA}

Estados Unidos fue el nuevo centro hegemónico a partir de la finalización de la Primera Guerra Mundial mientras que Europa se convirtió en la periferia. En el contexto europeo, España había sido un país que había evolucionado un poco al margen de los otros países europeos, en parte por su lento desarrollo industrial y por haberse declarado neutral en la gran guerra que le sirvió para sacar beneficios comerciales durante los 4 años de duración. En los años 20, esos beneficios no fueron aprovechados porque los empresarios españoles liquidaron los beneficios adquiridos en la década anterior al hacer políticas empresariales a corto plazo, protegidos por la dictadura de Primo de Rivera. Una vez finalizada la dictadura, la crisis del 1929 afectó a las empresas españolas debido a un comercio interior pequeño y empobrecido que estaba inmerso en una situación política incierta y por la crisis internacional. El número de sociedades constituidas durante la crisis, desde 1921 hasta 1931, fue de 11059 empresas con un capital de 5939 millones de pesetas, un número muy similar al decenio anterior, ya que el capital acumulado aún estaría impulsado por la gran guerra y por el ultra proteccionismo de la Dictadura de Primo de Rivera. En la etapa republicana, las sociedades constituidas desde 1932 a 1935 siguieron disminuyendo, reduciéndose a 3989 sociedades con un capital de 1818 millones de pesetas (Tafunell, 2005). 
En el caso de la industria catalana, la industria más influyente de España, y en concreto, la de Barcelona, se puede observar como las empresas constituidas entre 1929 y 1935 fueron poco rentables. Estas sociedades, según Carreras y Tafunell (2010), se alimentaron entre 1923 y 1929 de la etapa primorriverista que tendió a la inflación. Por esta razón, cuando se acabó la protección de la dictadura, las empresas primorriveristas se hundieron, demostrando ser entidades poco sólidas ya que su sistema financiero estaba sometido a la constante inversión de los accionistas y no a sus propias ganancias, por ello una vez que la inversión disminuyó, la rentabilidad de las empresas barcelonesas descendió notablemente.

Los estudios de Antoni Estevadeordal (1986) muestran que entre 1929 y 1935 existió una disminución del capital asociado en la industria catalana. El número de empresas constituidas en Barcelona descendió de 1850 entre los años 1921-1925 a 775 entre los años 19311935. En esta etapa, la pequeña y mediana empresa se autofinanció, mientras que las grandes empresas que habían sido promovidas por grupos bancarios actuaron de bancos industriales.

En Cataluña, la banca catalana agravó la situación de la empresa, dando lugar a dificultades que fueron desde el inicio estructurales. El nivel de especulación de la banca catalana en esos años, según se recoge en los Boletines Oficiales de Barcelona, llegó al 160\% en 1929, año en el que el crac de la Bolsa de Nueva York restringió los créditos existiendo con ello una baja en las cotizaciones en la bolsa. Este descenso estuvo contextualizado por los datos negativos de las exportaciones e importaciones en el territorio español entre 1928 y 1935 (un $-7 \%$ en los valores corrientes, un $-6,4 \%$ en las exportaciones y un $-2,1 \%$ en las importaciones referente a los precios constantes) respecto a los datos positivos que se habían registrado entre 1920 y 1928 (un 11\% en los valores corrientes, un 2,7 en las exportaciones y un 4,2 en las importaciones referente a los precios constantes), seguramente reforzado por su condición neutral en la guerra (Pinilla, 1994). A partir de los años 30, entre 1928-1935, existió un declive de la rentabilidad y la inversión de las empresas españolas (Carreras y Tafunell, 2010), en el que existieron 500 o 600 disoluciones por la insuficiencia de un mercado internacional de carácter proteccionista (Aranda, 2018a). El proteccionismo internacional existente no permitió que mercados periféricos en lento desarrollo como el español crecieran y desencadenó el aumento de problemas salariales y laborales, aunque previamente estas condiciones se vieran aliviadas por la coyuntura favorable de la Primera Guerra Mundial (Aranda, 2018a).

Una hipótesis para esta situación es que el excedente de producción almacenado le pudo restar utilidad al producto creado, lo que provoca que a largo plazo sea innecesario su propia producción, lo cual le restó sentido a una venta racional del producto en el mercado por la pérdida de su rentabilidad. En un proceso de fabricación febril dentro de un mercado inadecuado, la empresa puede advertir como su estrategia empresarial se ve afectada al no poder dar viabilidad a los distintos productos fabricados, lo que puede llevar a la compañía a realizar dos políticas económicas y laborales como diversificar su producción aumentando de manera constante su productividad, como fue el caso de la España Industrial, o iniciar políticas de recortes salariales $y$ despidos selectivos de personal para hacer viable la estructura de la empresa, que afectará posiblemente al valor del producto fabricado en relación con su consumo a largo plazo. Estas estrategias serían el punto de origen de la inestabilidad empresarial de una región o país, una enfermedad contagiosa difícil de remediar porque las diferentes tácticas sociales y financieras de tipo empresarial de la compañía ofrecen una dificultad extrema a una solución global.

Un excedente de producción conlleva a una situación financiera peligrosa de la empresa porque acarrea una estrategia crediticia o una necesidad de reunir capital que expone a la sociedad en una situación de riesgo, $y$ ante el riesgo, la tendencia general es que haya una disminución de la inversión y un crecimiento de la especulación en todos los sentidos dentro de la política social y económica de la empresa que se puede magnificar a gran escala. En el análisis de una empresa del sector algodonero 
fundada en 1847, llamada La España Industrial, permite ejemplificar como el excedente de producción en sus almacenes desencadenó una crisis financiera en la empresa, como se observa en una serie de balances de situación entre 1929 y 1933 (tabla 1).

TABLA 1

BALANCES DE SITUACIÓN DE UNA EMPRESA ALGODONERA “LA ESPAÑA INDUSTRIAL” PERIODO DE 1929-1933

(PESETAS)

\begin{tabular}{|c|c|c|c|c|c|}
\hline & 1929 & 1930 & 1931 & 1932 & 1933 \\
\hline LOCAL (URQ.) & 1654129 & 1639686 & 1622242 & 1604799 & 1587355 \\
\hline F. SANTS & 1488749 & 1491657 & 1533831 & 1508465 & 1485132 \\
\hline F. SABADELL & ----- & ----- & 350000 & 346500 & 393096 \\
\hline MAQ. SAB. & ----- & ------ & 989867 & 1001846 & 994166 \\
\hline MAQUINARIA & 4038766 & 3906199 & 4358870 & 4312236 & 4285063 \\
\hline M. ELÉCTRICA & 140584 & 136472 & ---- & 128249 & 124.137 \\
\hline GASTOS & 228439 & 242814 & 228315 & 240389 & 298670 \\
\hline MERCANCÍAS & 4086029 & 5085749 & 6993389 & 6949171 & 6160844 \\
\hline "DEUDORES" & 2786435 & 2899984 & 4044334 & 4834986 & 5521445 \\
\hline CAJA & 1588458 & 1548589 & 316341 & 1331211 & 1938369 \\
\hline ACTIVO & 18113223 & 18191738 & 22397202 & 23616616 & 23691481 \\
\hline CAPITAL & 8000000 & 8000000 & 10000000 & 10000000 & 10000000 \\
\hline ACREEDORES & 450469 & 1327954 & 2000023 & 831858 & 444707 \\
\hline DEUDAS & 32994 & 34794 & 163362 & 44708 & 123418 \\
\hline ACCIONISTAS & 585702 & 429640 & 253446 & 177036 & 198.996 \\
\hline BENEFICIOS & 1795453 & 1275472 & 1191164 & 1164644 & 1519457 \\
\hline FONDOS RES. & 800000 & 800000 & 2530500 & 2532500 & 2532000 \\
\hline SEGUROS & 6448603 & 6323877 & 6223877 & 6323877 & 6323877 \\
\hline PASIVO & 18113223 & 18191738 & 22397202 & 23616616 & 23691481 \\
\hline
\end{tabular}

Fuente: Elaboración propia a partir del Archivo Nacional de Cataluña (s.f), Balances de situación, fondo de la España Industrial, 1929-1933.

Los directivos de la sociedad España Industrial a finales de 1928, no pudieron ocultar que la empresa estaba en crisis porque los beneficios no cubrían los gastos. Esto se solucionó en mayor medida por el aumento de capital social y de las líneas crediticias, aunque autores como Enric Ribas Mirangels (1999) señalan que esta situación se compensó gracias a una emisión de bonos. Por ello, en 1929, se hizo un primer balance de situación, al que siguieron otros hasta a 1933, inclusive.
En estos balances de situación, se pueden conocer las características de la crisis que atravesó una empresa como La España Industrial en esos años existiendo un equilibrio entre el activo y el pasivo de la empresa. En el balance de la empresa, se contabilizó, en 1929, un activo-pasivo de 18113223 pesetas que aumentó de manera progresiva hasta la cantidad de 23691481 pesetas en 1933.

En el activo fijo, la empresa España Industrial demostró poseer un valioso patrimonio 
inmobiliario englobado en la fábrica de Sants a la que se le añadió la sede de plaza Urquinaona en 1924 y la fábrica de Sabadell en 1931. Este valor patrimonial fluctuó entre las 3142878 pesetas de 1929 (sin la fábrica de Sabadell) hasta las 3465583 pesetas con la fábrica de Sabadell en funcionamiento. Otro importante valor fijo de la empresa fue la maquinaria, cuyo valor osciló entre las 4038766 pesetas de 1929 a las 4285063 pesetas en 1933, sin que hubiera en aquellos años muchas diferencias. El valor de la maquinaria fue muy exiguo en comparación con el valor total de la maquinaria de la sociedad. En el activo circulante de la empresa, el valor de las mercaderías aumentó de 4086 029 pesetas en 1929 a las 6160844 pesetas en 1933, y hubo un repunte en 1931 de 6993389 pesetas. En el activo realizable, el costo de los deudores aumentó de las 2786438 pesetas a las 5521445 pesetas. En la caja, la empresa se mostró aparentemente saludable pues aumentó de las 1588458 pesetas a las 1938369 pesetas.

En ese periodo existió un aumento de las líneas de crédito como la incorporación del Banco Zaragozano y el Banco Arnús, este último dedicado a los valores bursátiles. En el pasivo exigible a corto plazo o fondos ajenos a corto plazo, el costo de los acreedores osciló entre las 450469 pesetas de 1929 a las 2000 023 pesetas, para volver a disminuir en 1933 a las 444707 pesetas. Las deudas contraídas fueron exiguas, entre las 32994 pesetas en 1929 a las 123418 pesetas en 1933, posiblemente porque fueron absorbidas por los beneficios de la empresa, que disminuyeron de las 1795453 pesetas en 1929 a los 1191164 en 1931 como punto más bajo, para aumentar a las 1519457 pesetas en 1933.

En el pasivo a largo plazo destacó la emisión de bonos al 6\% que apareció en 1932 para contrarrestar las deudas a corto plazo $y$ solventar de una manera momentánea la crisis financiera de la empresa. En la masa patrimonial del neto destacó que el capital social aumentó en 1931 de ocho a diez millones y que, por el contrario, el valor de las acciones se fue empobreciendo de las 585702 pesetas en 1929 a las 198996 pesetas en 1933. El fondo de reserva de la empresa pasó de las 800000 pesetas en 1929 a dividirse en fondo de reserva especial en el que había 1532000 pesetas en 1933 y un fondo estatutario en el que se depositó un 1000000 de pesetas a partir del 1930. La España Industrial poseía un fondo que cubría posibles accidentes cuyo valor osciló entre las 6 448603 pesetas en 1929 a las 6323877 pesetas de 1933 (Aranda, 2018b).

En estos balances se muestra que en esta etapa existió una serie de beneficios exiguos. Los pobres beneficios hicieron aumentar el valor de la producción, el valor de las materias primeras acumuladas y el valor de las piezas elaboradas, lo cual dificultó la comercialización de los productos de la empresa debido a un mercado interior empobrecido $y$ al proteccionismo comercial en el mercado internacional (Aranda, 2018b). La producción a pesar de esta situación no se detuvo y, posiblemente, el desarrollo tecnológico tuvo incidencia en ello. La introducción de la electricidad a partir de 1913 en la empresa provocó que las viejas estructuras y las nuevas convivieran, aunque no fuera sin que hubiera problemas. La poca capacidad de regulación de la velocidad en los sistemas eléctricos en las diferentes máquinas $y$ la excesiva potencia provocaba problemas en los generadores. Según los planos de los diseños de algunas de las máquinas que existían en la empresa - guardados en el Museo de la Estampación de Premià de Mar-, estas dificultades se intentaron paliar con una serie de sistemas o engranajes. Asimismo, se utilizó el sistema de la polea-correa.

El uso de múltiples poleas permitió obtener una gran ventaja mecánica, ya que llevaba grandes pesos con poco esfuerzo. Este método se combinó con otras máquinas que se basaron en un sistema de engranaje-correa. Este método permitió mantener la relación de transmisión constante, incluso, transmitiendo grandes potencias entre los ejes, que se tradujo en una buena eficiencia mecánica. Asimismo, permitió conectar ejes que se cruzaban o que se cortaban, con lo cual su funcionamiento pudo llegar a ser muy silencioso, pero a su vez tenían un alto coste $y$ poca flexibilidad (Aranda, 2018b).

La empresa con la electrificación combinó el motor de combustión con el eléctrico. 
Los motores eléctricos tenían un tamaño y peso más reducido, además eran máquinas reversibles pudiendo operar como generadores, convirtiendo con ello la energía mecánica en eléctrica. Su inconveniente era su alto coste en el mantenimiento $y$ que eran incapaces de funcionar a velocidades bajas, sobre todo en el arranque. Entre 1929 y 1935, se detectó una bajada de la rentabilidad financiera en muchas de las empresas barcelonesas por un exceso de productos acabados $y$ materias primas almacenadas en los respectivos almacenes. La hipótesis que se plantea es que este exceso vino causado por el paulatino cambio del carbón por la electricidad y que provocó que se intensificara la productividad de las empresas y se acelerara la velocidad (v) de la propia producción (P). Un exceso de la producción solo se puede entender si se incluye al valor del trabajo (trab), el factor tecnológico de las máquinas (tec) y la electrificación (E) como principios de los costes financieros de las empresas, que en su conjunto modificaron los valores del sistema productivo (Sp). Es decir, la velocidad es la que influye $y$ sufre las consecuencias de los valores del trabajo, integrados en la DG (demanda global) que es igual a C (consumo) + I (inversión) + G (gasto público) + Ex (exportación).

El valor del trabajo realizado se ajusta dentro de la demanda global como parte de los costes, en los que estos aumentarán o disminuirán condicionados por la energía empleada. Si se calcula el sistema productivo a partir de la pequeña diferencia existente entre unidades de medida de potencia como es el caso de $\mathrm{CV}$ (caballos de vapor) y HP (horsepower). Al comparar los sistemas productivos antes y después de la introducción de HP, se observa que la variable $\mathrm{Vi} / \mathrm{Vj}$ es la que rige el propio sistema, en el que la velocidad (v) es igual a la distancia/tiempo, ya que este está incluido en la definición de velocidad y es un parámetro inversamente proporcional a la misma. Esto quiere decir que, si la velocidad aumenta, es porque el tiempo, que hace de dividendo en la ecuación, disminuye. De igual modo, si la velocidad aumenta, es porque la distancia aumenta, ya que la distancia a diferencia del tiempo, es directamente proporcional a la velocidad.
En referencia a esto, se puede concretar que si el caballo de vapor (cv) como potencia es una variable que tiende a 1 , si tomamos como referencia las unidades internacionales (UI), esta hará que el sistema productivo (Sp) disminuya, en cualquiera de los casos. Sin embargo, con HP esta variable tiende a 0,98 (también siguiendo el patrón de UI) con lo cual, el Sp se verá aumentado.

La entrada de la electrificación provocó que el factor fuerza (Ff) disminuyera, ya que a más velocidad adquirida menos capacidad de regulación ha de poseer la tecnología, siendo el momento en que el factor humano ha de controlar este desequilibrio, pero al no ocurrir se producen ciclos febriles en la producción (Aranda, 2018b). La clave de esta exuberancia en la fabricación se produjo porque la velocidad (v) siempre es constante, no aminora, independientemente de la tecnología, si acaso la acentúa por la inercia adquirida.

Esta inercia se hace observable a partir de las sobrecargas y cortocircuitos que acaban quemando transformadores $y$ alternadores, como se describe, por ejemplo, en los diarios semanales de la España Industrial entre 1914 y 1918, guardados en el Museo de la Estampación de Premià de Mar. En el circuito, una sobrecarga se produce cuando a este se le añaden cargas que no están previstas para que el sistema les pueda suministrar la corriente necesaria para su funcionamiento. A medida que se va agregando cargas al circuito, el consumo de corriente aumenta y, por ello, el coste. Esta sobrecarga se produce cuando existe un camino de baja resistencia por donde puede circular la corriente, si la resistencia baja, existe un aumento drástico de la corriente eléctrica. Esta relación se puede confirmar cuando existen dos tipos de sistemas generales de alimentación como el sistema de corriente directa (positivo y negativo) y el sistema de corriente alterna (potenciales y neutro). Es entonces cuando se produce el cortocircuito, ya que entran en contacto dos o más de estas líneas de alimentación en un circuito (Aranda, 2018b).

Este aumento de la corriente se completa a través de la ley de Ohm que desarrolla la teoría de que en un circuito cerrado, el voltaje, 
la corriente eléctrica y la resistencia deben tener valores debidamente controlados para un buen funcionamiento del sistema. Una condición para que haya un cortocircuito queda determinada al eliminarse desde el punto de vista práctico, la resistencia de consumo del circuito, ya que, si la resistencia disminuye aproximadamente a cero, la intensidad de la corriente tiende a infinito $y$ afecta, sobre todo, a los motores monofásicos y trifásicos.

La teoría que se plantea es que a principios del siglo xx, la electricidad aceleró la producción industrial acortándose los plazos de entrega y venta por culpa del cambio energético, sin que se redujeran de manera eficaz los costes, lo que desequilibró el sistema financiero de las empresas que utilizaron la tecnología no como un medio de desarrollo a nuevas vías de fabricación, sino como modo de reducir la carga de gastos de las compañías, la mayoría con el fin de controlar y reducir la mano de obra o la carga salarial. Esto afectó a las economías domésticas de los trabajadores y a las finanzas de la propia empresa. Todos estos procesos hicieron aumentar la producción de la empresa de manera constante y radicalizaron los patrones de contratación de mano de obra no cualificada y barata. La empresa aumentó su patrimonio con la adquisición de la Fábrica de Sabadell en 1931 como defensa financiera para compensar los costes en los cuales la tecnología condicionó la producción, lo cual afectó a la estructura laboral de la empresa La España Industrial, tanto a nivel salarial como en la forma de entender el trabajo.

La única manera de dar lógica al efecto multiplicador-acelerador (a más inversiónmayor consumo) de la teoría Keynesiana, es a partir de la velocidad a la hora de producir analizando el excedente de producción, al entender su relación con la saturación comercial como un síntoma que provoca que el sistema económico y social decline, lo cual se presenta como una enfermedad que se transmite a través de la inestabilidad empresarial de múltiples formas (Aranda, 2018b). Ramón Soler Becerro (2012), quién estudió sociedades como la Fábrica de la Rambla de Vilanova o la Colonia Sedó, señaló que en el primer caso, los recursos a corto plazo de la compañía fueron muy limitados y se estaba ahogando por el aumento de acreedores, por esta razón, entre 1929 y 1935, la empresa tuvo que cerrar para agotar los excedentes de producción. En el caso de la Colonia Sedó, los accionistas hicieron un esfuerzo para equilibrar los posibles déficits financieros para absorber la acumulación de la producción (Soler, 2012). No existió ni existe una única forma de actuación por parte de las empresas en una etapa crítica, pero el excedente de producción es un síntoma que se ha de tener en cuenta al considerar a la velocidad como fondo de cuestión. Una empresa en un contexto de crisis tiene que nivelar, por el bien de su estructura, la creciente aceleración de la producción, resultado de la innovación tecnológica con el poco equilibrio laboral, ya que su mala gestión es la que crea finalmente las diferentes desigualdades económicas y sociales.

Una de las ideas de F. Hayek era que el aumento de stock de bienes de capital no siempre es positivo, ya que la inversión puede ser excesiva y estar basada en proyectos de mala calidad financiados por una política crediticia especulativa que no estaba sustentada en un ahorro voluntario de la sociedad. Este proceso produciría grandes niveles de sobreproducción, el exceso de oferta de unos bienes de consumo $y$ de capital que la gente no puede o no quiere comprar. Es fundamental que la rigidez de las instituciones no fuera tal para que los salarios reales se ajustaran a la oferta del mercado correspondiente (Feito, 2008).

Los ciclos recesivos desde 1929 han sido cada vez más frecuentes, pero las dos crisis, la de 1929 y la de 2008, coinciden en que la Gran depresión de los años 30 finiquitó la primera etapa de la globalización por culpa de la demanda creciente de proteccionismo y por una creciente hostilidad hacia la inmigración en los países receptores (James, 2003). Estos problemas se extendieron globalmente por un sistema que había impuesto una serie de exigencias cada vez mayores, fracasando finalmente porque los países de entonces y sus instituciones, no supieron manejar las consecuencias psicológicas de un mundo interconectado, lo que provocó que entre 1920 y 1930, acabara por destruirse el 
sistema global, y el mundo retornase a las administraciones económicas nacionales autárquicas o casi autárquicas (James, 2003).

La crisis de 2008 ha puesto en duda al sistema global existente porque la política de los países desarrollados, con la aparición de partidos políticos ultraconservadores, se ha vuelto proteccionista, lo que ha dificultado las relaciones comerciales entre las respectivas naciones y con una conducta hostil hacia las oleadas migratorias de hombres, mujeres y niños, quienes buscan una vida más estable.

El exceso de crecimiento del comercio de mercancías en los países desarrollados ha provocado que la economía de los países en vías de desarrollo se haya ralentizado por las dificultades de crear una red empresarial suficientemente sólida y un negocio local próspero, debido a la incapacidad de ser competitivos en un mercado sobresaturado por los países desarrollados que pone en cuestión que el mundo global existente sea en la práctica real y efectivo.

\section{EL COMERCIO AL POR MENOR Y EL COMERCIO} AL POR MAYOR COMO OBJETO DE ESTUDIO DE LA CRISIS DEL 2008. LA SITUACIÓN DE ESPAÑA

El informe estadístico del comercio mundial 2018 (Organización Mundial del Comercio, 2018) expone que el comercio mundial registró en 2017 su mayor tasa de crecimiento en 6 años en términos de volumen. El volumen del comercio de mercancías, medido por el promedio de las exportaciones y las importaciones, creció en un $4,7 \%$, lo que supuso el primer incremento anual superior al 3,0\% desde 2011 . El crecimiento del volumen del comercio fue sólido en 2017, pese a las tensiones comerciales. Durante el primer semestre de 2018, esas tensiones se tradujeron en la aplicación de una serie de medidas restrictivas del comercio que abarcaban una amplia gama de bienes y grandes economías, debido al riesgo de que en 2019-2020, se desencadene un ciclo de retorsiones que podría perturbar el comercio y el crecimiento mundial (OMC, 2018).

El incremento del volumen del comercio en 2017 fue del 4,7\%, situándose cerca de la tasa media del 4,8\% registrada desde 1990 , y estando muy por encima de la media del 3,0\% posterior a la crisis. El acrecentamiento del comercio de mercancías en volumen registrado se debió en parte a la debilidad del comercio durante los 2 años precedentes, lo que estableció una base de referencia más baja para la expansión actual y además, se tiene que advertir el hecho de que en 2017, el crecimiento del comercio en términos de valor fue más sólido que en términos de volumen. Esto se reflejó tanto en el aumento de las cantidades como en la subida de los precios.

Las estimaciones del aumento del PIB se registraron al alza durante 2017, lo que explica en parte que el crecimiento del comercio de mercancías del 4,7\% para ese año superara incluso las previsiones anteriores más optimistas, ya que se realizaron sobre la base de las proyecciones económicas existentes a principios de 2018, en general positivas, que reflejaron, no solo el incremento de la inversión y del empleo, sino también un aumento de la confianza de las empresas y los consumidores. Sin embargo, varios riesgos a la baja pudieron socavar fácilmente esta situación a partir del 2019. A pesar de la mejora del crecimiento del comercio, siguen presentes algunos factores estructurales que han afectado al comercio en los últimos años, entre ellos: 1) el proceso de reequilibrio de la economía china, 2) de la inversión (que tiene un contenido de importaciones muy elevado) al consumo (con un contenido de importaciones menor que la inversión), y 3) el paulatino descenso del ritmo de la apertura del comercio mundial en los últimos decenios.

El posible reequilibrio de China podría frenar ligeramente las importaciones a corto plazo, pero debería generar un mayor crecimiento sostenible a largo plazo, lo que estimularía el comercio, sino fuera por la guerra comercial existente en 2019 entre China y Estados Unidos, o el Brexit, que podría generar la ausencia de una mayor apertura del comercio, lo que podría provocar que el crecimiento de los intercambios comerciales fuese menor, tanto a corto como a largo plazo (omc, 2018).

En el sur de Europa, específicamente en España, se muestra, por ejemplo, como el comercio al por menor en el periodo posterior 
a 2008, en concreto entre el 2013 al 2018, fue inferior al comercio al por mayor.

El comercio al por menor en el año 2013 fue en el cuarto trimestre del $-3,1 \%$ y aunque aumentó en años posteriores, mostrándose constante a partir del cuarto trimestre de 2014 con un $2,1 \%$, en 2015 con el 1,9\%, en 2016 con un $2,3 \%$ y en el 2017 con un 1,4\%, en el segundo trimestre del 2018 volvió a caer con el -1,7\%. Estos datos van unidos al déficit comercial que según el Ministerio de Industria, Comercio y Turismo, alcanzó en noviembre de 2018 los 30 592,6 millones de euros, un $34,8 \%$ superior que el registrado en el mismo periodo de 2017 con un déficit de 22694,2 millones de euros (gráfico 1).

\section{GRÁFICO 1 \\ ENCUESTA COYUNTURAL SOBRE STOCKS Y EXISTENCIAS COMERCIO AL POR MENOR \\ ESPAÑA, 2013-2018}

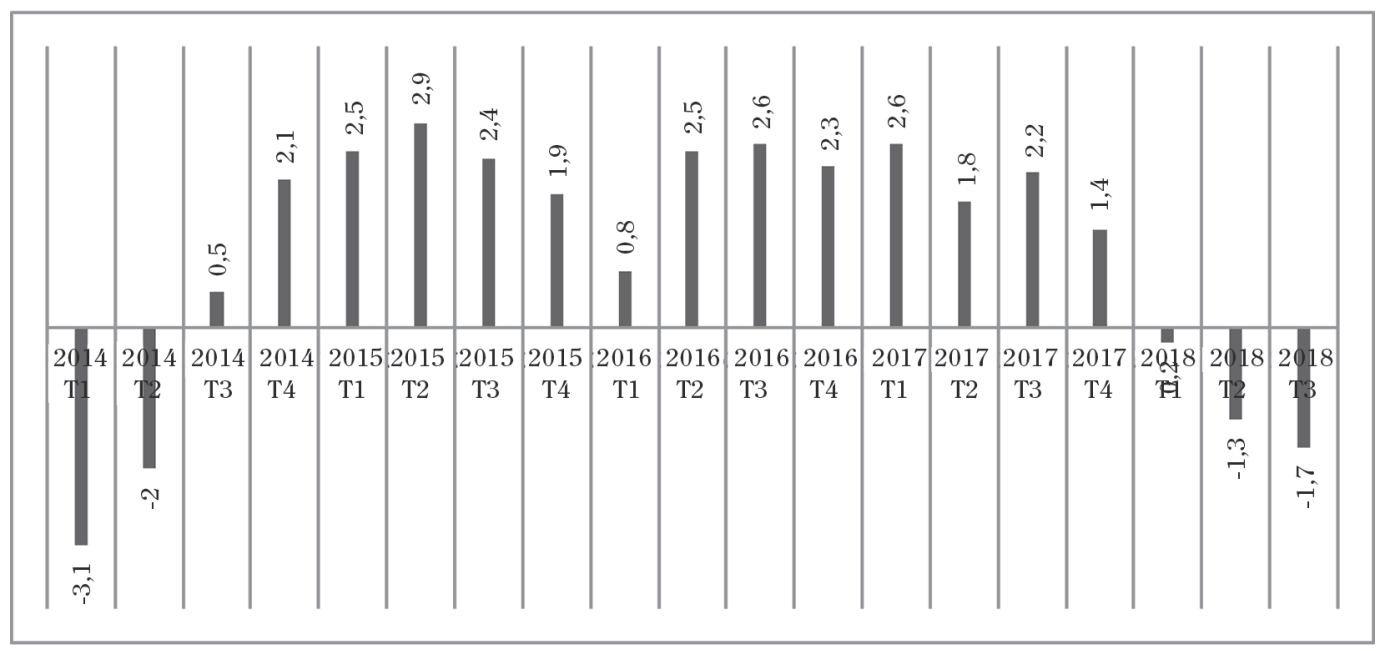

Fuente: Instituto Nacional de Estadísticas, 2013-2018.

La tasa de cobertura se situó en el $89,6 \%$, es decir, 2,2 puntos porcentuales menos que la de enero-noviembre de 2017 $(91,8 \%)$. El saldo comercial no energético arrojó una deuda de 7645,9 millones de euros con un pérdida de 3424,8 millones de euros en enero-noviembre de 2017, mientras que el déficit energético de la balanza comercial energética se incrementó un 19,1\%.
En comparación con los resultados acumulados para España, estos datos a nivel internacional muestran un menor crecimiento de las exportaciones en relación con los principales socios. Las exportaciones de la UE-28 crecieron un $5,1 \%$ interanual, las de la zona euro un $4,9 \%$, las de Alemania un $3,7 \%$, las de Francia un $4,2 \%$, las de Italia un $3,5 \%$ y las Reino Unido con un 7,4\% (gráfico 2). 


\section{GRÁFICO 2 \\ ENCUESTA COYUNTURAL SOBRE STOCKS Y EXISTENCIAS \\ COMERCIO AL POR MAYOR EN ESPAÑA \\ 2013-2018}

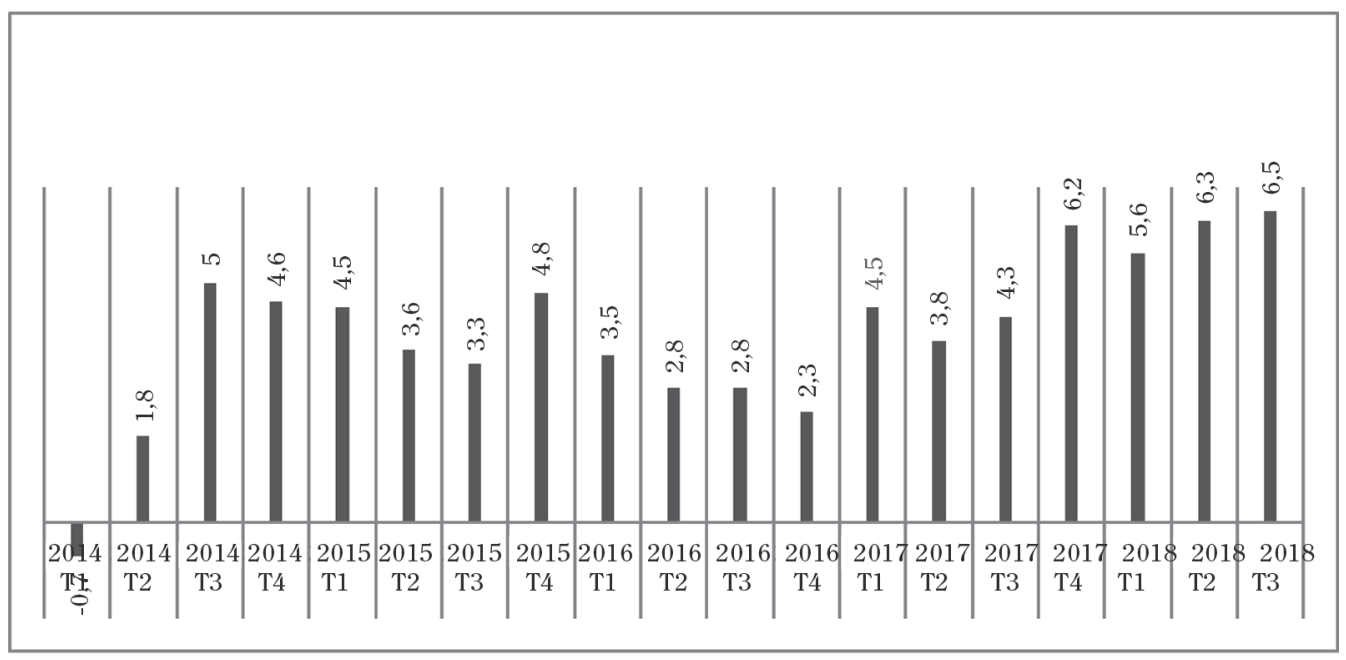

Fuente: Instituto Nacional de Estadísticas, 2013-2018.

El comercio al por mayor en el primer trimestre de 2014 fue del $-0,7 \%$, sin embargo, este aumentó en el cuarto trimestre del 2015 con un $4,8 \%$, en el 2016 con un $2,3 \%$, en el 2017 con el 6,2\% y el tercer trimestre de 2018 con el $6,5 \%$. El comercio mayorista es una forma de comercio en el que se compran y se almacenan en grandes cantidades, al venderse en lotes de una cantidad designada a los revendedores pero no a los consumidores finales.

Las desventajas de este tipo de comercio es el encarecimiento de bienes, la reducción de los niveles de rentabilidad de la producción y una minimización del poder de decisión, ya que el comercio mayorista está por encima del cumplimiento de los planes estratégicos de las empresas que al adquirir una gran cantidad de productos, estos se almacenan produciéndose problemas de stock, como por ejemplo, la obsolescencia, problemas de flujo de efectivo, una menor rentabilidad, una reducción de los márgenes de ventas o hasta dificultades tributarias que exponen a las empresas al contexto político y económico del país en el que reside. En concreto, el caso de España es el de un país con graves problemas de de-sempleo, precariedad laboral y salarial que lo expone a padecer una crisis económica similar a la que padeció en el 2008, al producirse una oferta excesiva sin que haya una demanda real que la pueda consumir con coherencia.

El estudio y una buena gestión de los inventarios son el factor que puede dar un sentido real, sea en 1929 o en el 2008, al Kaos económico, un caos que basado en el exceso de producción acaba afectando a la estructura empresarial de un país y a la postre a la sociedad que la forma (gráfico 3). 
GRÁFICO 3

EVOLUCIÓN ANUAL DEL NÚMERO DE EMPRESAS CONCURSADAS EN ESPAÑA

2005-2017

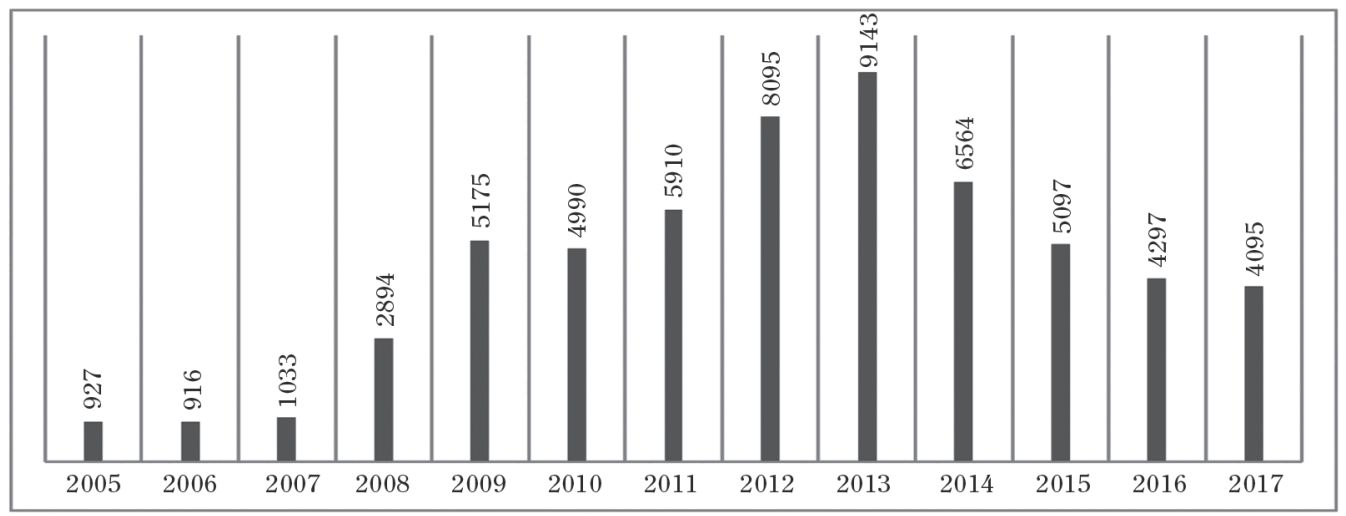

Fuente: Instituto Nacional de Estadísticas, 2005-2017.

En España, el número de empresas en concurso de acreedores a partir del 2008 creció de las 2894 hasta las 9143 en el 2013, disminuyendo hasta las 4095 empresas en el 2017. Axesor, la agencia de rating española, publicó que entre el 2008 y el 2015 más de 45000 empresas se declararon en quiebra en los últimos 8 años. Es decir, de media, cada día más de veinte empresas entraron en quiebra desde 2008. El cálculo de 2015 fue de 5115 nuevos concursos, que con todo supuso una regresión del 21,4\% sobre el año anterior. El grueso de estos procesos formales de insolvencia se concentró en los últimos 4 años, que con 28195 incidencias, representó el $62,3 \%$ de todos los concursos iniciados entre 2008 y 2015 (La crisis se salda con 45.000 empresas españolas en quiebra, 13 de enero de 2016).

La pauta de mortalidad empresarial en España en el año 2015 fue de un 8,1\% según el INE, reduciéndose en un punto y medio porcentual en 2017, respecto a la que hubo en el peor año de la recesión (2012), pero a pesar de ello, se estaba dos puntos por encima de los datos anteriores al estallido de la crisis en 2007. La rotación corporativa fue tan alta que de las 76844 empresas creadas en 1992, solo 16589 permanecieron activas en el 2017, apenas el $22 \%$, según un estudio presentado en su momento por Informa D\&B (Fernández, 12 de mayo de 2018).

Las grandes marcas como Mercadona, Zara, H\&M, Primark, Uniqlo, Fnac o Mediamarkt, se han adueñado del centro de muchas ciudades, lo cual ha perjudicado a muchos pequeños comerciantes $y$ antiguos centros comerciales, quienes han tenido que cerrar. Como consecuencia de ello, la quiebra de empresas repuntó en julio de 2018 dando un paso atrás. El número de concursos de acreedores se disparó al 25,7\% en el séptimo mes del año, con 430 procesos concursales abiertos, la cifra más alta desde 2014 en un mes de julio. El sector comercial arrastró una dinámica negativa desde principio de año. El número total de concursos de acreedores entre enero y julio fue de 2627 , un $0,6 \%$ menos que en los mismos 7 meses de 2018 . Pero en el comercio, hasta julio fue negativo, con un repunte del 8,9\%, hasta los 627 procesos (La crisis del comercio tradicional dispara la quiebra de empresas, 02 de agosto de 2018).

El negativo crecimiento comercial y empresarial ha provocado el crecimiento de la deuda pública. La deuda en los últimos años se ha mostrado como uno de los principales capítulos de los presupuestos generales del Estado. 
El pago de intereses de la deuda se ha duplicado con creces en los últimos 10 años en el que ha pasado de los 15265 millones de euros en el año 2008 a los 31547 millones de euros en el 2018. Esta partida supuso el 8,9\% del presupuesto de ese año (Relaño, 01 de setiembre de 2018). Según el Instituto Nacional de Estadística, en el año 2017, el resultado de esta deuda fue que cada ciudadano debía de media unos 24589 euros. Esa cifra superaba, por ejemplo, los 23106 euros en los que ine situaba el sueldo medio, es decir que cada ciudadano en España tendría que dedicar el 110\% de ese salario para abonar el pasivo público, que por persona asciende a 25494 euros al año, según datos del Observatorio de Deuda que publica trimestralmente la Autoridad Fiscal (AIReF) (Viaña, 17 de febrero de 2018).

En paralelo a estos datos, el gasto de los hogares no dejó de crecer. El ahorro doméstico estuvo bajo mínimos, mientras el recurso a la deuda fue cada vez mayor. La banca por su parte, hambrienta de rentabilidad, redobló su apuesta por el préstamo al consumo, con tipos cercanos al 8\%. La concesión a las familias de créditos en 2017 para este tipo de gasto alcanzó los 43148 millones, el mayor aporte de financiación en una década. Más, incluso, que lo destinado a nuevas hipotecas (Bolaños, 06 de febrero de 2018). Estas cifras van unidas al 14,3\% de tasa de desempleo que existió en diciembre de 2018 en España, siendo menor al $16 \%$ que existió en el año anterior pero superior a la media de la Unión Europea, ocupando el segundo lugar con la tasa de desempleo más alta tras Grecia.

\section{EL PROBLEMA COMERCIAL DE UN MUNDO GLOBAL CON TENDENCIA A LA DESIGUALDAD}

La producción en los llamados países en desarrollo, en general, estuvo desacelerando entre el 2008 y el 2018, según indica el índice de producción industrial interanual (IPI). Los países más industrializados en el 2009 disminuyeron de manera notable el ritmo de producción como fue el caso de Estados Unidos (-12,9\%), Alemania $(-18,2 \%)$ o Japón $(-28,4 \%)$. Entre el 2010 y el 2012, hubo una cierta recuperación, menos en Japón, cuya producción aumentó excepcionalmente (17,5\%). Este crecimiento volvió a disminuir a partir del 2013 hasta el 2018, siendo el país nipón un caso destacado, aunque el descenso o el leve crecimiento fue general.

El caso de China es excepcional. A partir de 2013, su descenso (menos en el año 2017), ha sido general. La producción en los países industrializados no se ha detenido, solo se ha diversificado por el globo. Estos datos regresivos tienen relación con la deslocalización industrial $y$ al desarrollo del comercio online, que en el caso de España, en 2017, estas transacciones de productos y servicios online alcanzaron una facturación de 24185 millones de euros, un $20,8 \%$ más que en 2015 , según el informe de la Comisión Nacional de los Mercados y la Competencia (CNMC), siendo la contribución del $e$ Commerce al Producto Interior Bruto (РІB) de los países del G-20 superior al 5\%.

El comercio online tiene como ventajas la capacidad para procesar una gran cantidad de pedidos con un coste bajo a nivel financiero y la utilización de pocos trabajadores útiles, sin embargo, son empresas poco estables por la alta competitividad que existe en los diferentes mercados, creando y destruyendo empresas en poco tiempo, repercutiendo en los ciclos de bienestar de la sociedad del futuro, demostrando como la introducción de nuevas técnicas de fabricación $y$ distribución desde comienzos del siglo pasado han permitido el desarrollo de la sociedad, pero también la ha vuelto más inestable.

El comercio mundial retrocedió entre octubre y diciembre de 2018 un $0,9 \%$ trimestral frente al 1,3\% que creció en el trimestre anterior. El crecimiento que existió después de 2008 según datos del СРв World Trade Monitor, procedió al 43\% de las exportaciones entre el 2016 y el 2017, años de mayor crecimiento de los países en desarrollo, a pesar de que en general existió un difícil crecimiento del negocio local en beneficio de las grandes multinacionales, ya que la deslocalización de las empresas no ha representado para la población perteneciente a los países de acogida, en mejores salarios ni en mejores condiciones laborales. En el Informe Mundial sobre Salarios (Organización Internacional del Trabajo, 2017), se señala que en este tiempo 
existió una desconexión entre el crecimiento económico y el crecimiento de los salarios. Por esta razón, se reclama en dicho informe que hay que "impedir la aplicación de políticas de reajuste salarial en demasiados países al mismo tiempo o recortes salariales con desenlaces competitivos para desarrollar las exportaciones ya que puede ocasionar una caída de la demanda agregada, regional y mundial, o deflación" (pp. xxii-xiv).

La Organización Internacional del Trabajo (2018) detalla que las soluciones son: 1) la mejora del salario mínimo y la negociación colectiva, 2) desarrollar el crecimiento de la productividad de las empresas sostenibles y, 3) resolver la desigualdad salarial entre los diferentes grupos de trabajadores. Otras medidas serían nuevas medidas fiscales en forma de impuestos y transferencias para resolver la cuestión de los salarios y la desigualdad, mediante políticas con incidencia directa en los salarios y la distribución salarial como elementos importantes de una respuesta global. Según el informe del Doing Business (Banco Mundial, 2018), dentro de la zona del África Subsahariana existió poca facilidad para hacer negocio en la mayoría de los países de la zona como pueden ser el caso de Somalia, Eritrea, Sudán del Sur o hasta las islas Mauricio (tabla 2). 
El exceso comercial como causa de la crisis del mundo global en las depresiones de 1929 y 2008...

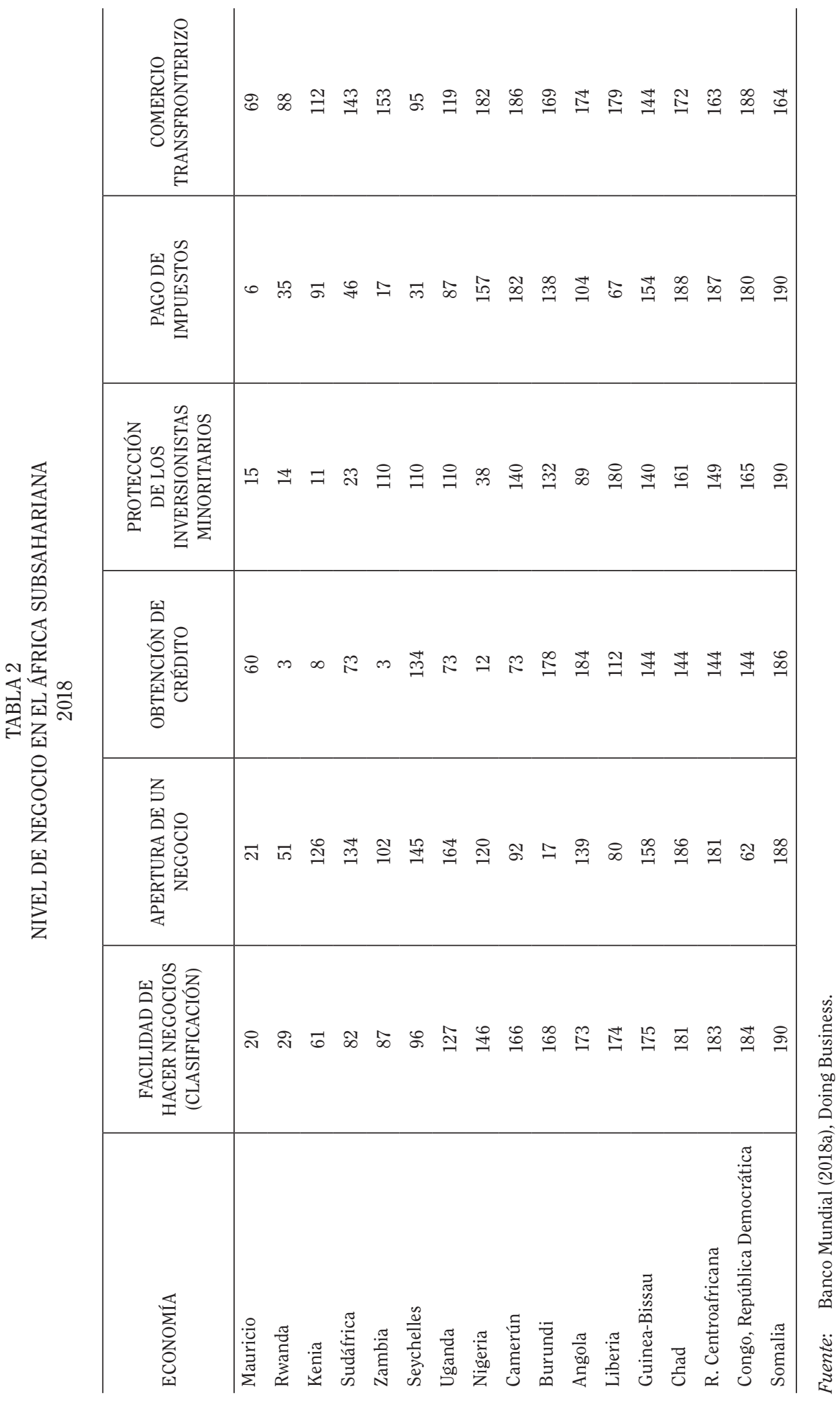


En la zona del África Subsahariana, se observa la poca protección estatal que tienen los inversionistas minoritarios del país y lo débil que es la administración por la dificultad al cobrar impuestos por parte del Estado, teniendo en cuenta aquellas zonas que son paraísos fiscales, distinguiéndose economías como la de Sudán (190), el Chad (181), Angola (173), Liberia (174), la R. Centroafricana (183) o Camerún (166). El Banco Mundial señaló que en el año 2019, el crecimiento económico de África al sur del Sahara continuaría recuperándose de manera continua. Se esperaba que llegara a 3,1\% en 2018, y promediara 3,6 \% en el periodo 2019-2020. Sin embargo, este moderado crecimiento sigue siendo desigual, con diferencias importantes entre los países (Banco Mundial, 2018 y 2019a) (tabla 3). 


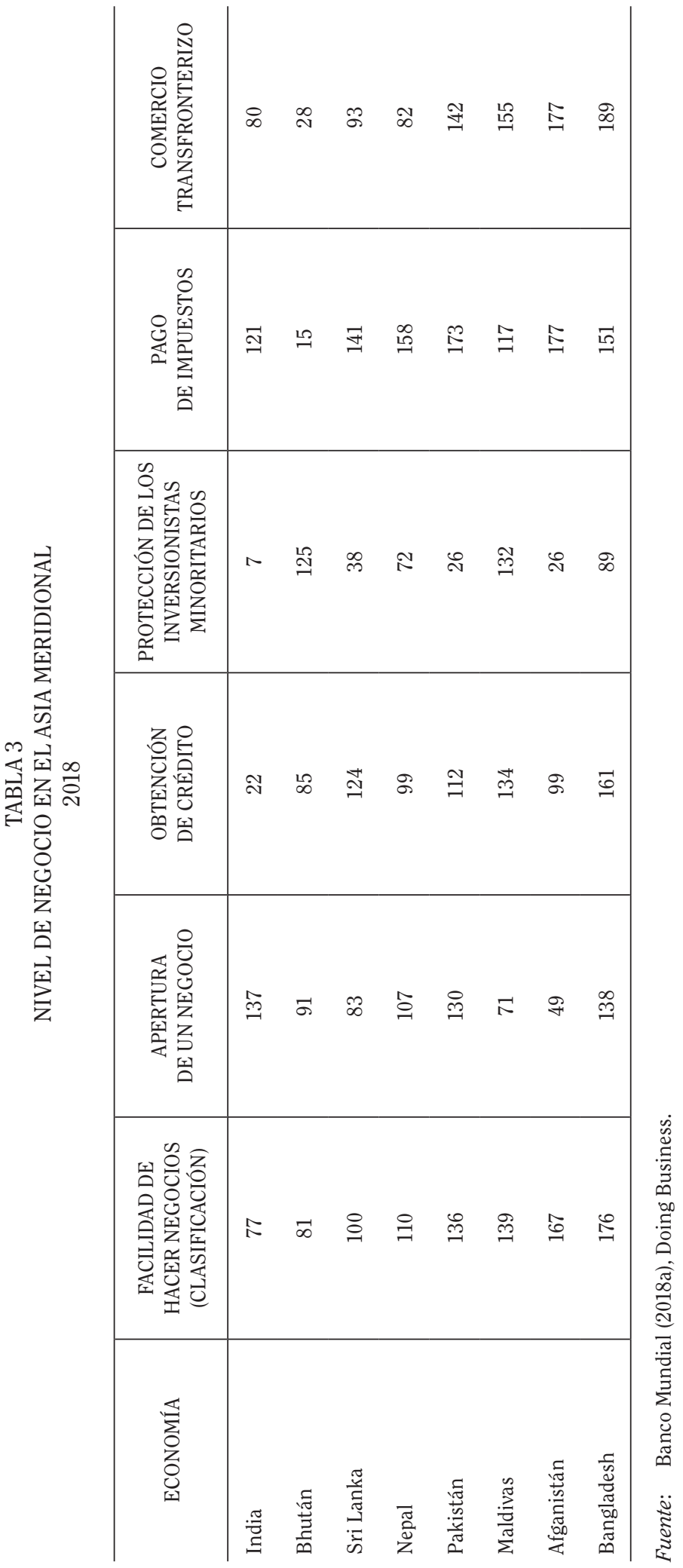


En la zona del Asia Meridional existen economías como la de India (77), Bhután (81), Sri Lanka (100), Nepal (110), Pakistán (136) o Bangladesh (176). Según el Banco Mundial, se esperaba que Asia meridional fuera la región de más rápido crecimiento del mundo en el 2019. Según las proyecciones, este crecimiento podría repuntar hasta llegar al 6,9\% en 2018, principalmente como consecuencia de la recuperación de India. El incremento debería fortalecerse y llegar al 7,1\%, en promedio, entre los años 2019-2020, reflejando "mejoras generalizadas en la mayor parte de la región. Asia meridional debería mantener su posición como la región de más rápido desarrollo e incluso superar a Asia oriental y el Pacífico"
(Banco Mundial, 2019b, párr. 2 y 3). Si bien, este pronóstico se mantenía prácticamente sin alteraciones desde enero de 2018, la tasa de crecimiento que se proyectaba era levemente menor, principalmente debido a una corrección a la baja en 2019 para Pakistán (Banco Mundial, 2019b). El Banco Mundial señaló que la zona de Latinoamérica y el Caribe creció un $0,6 \%$ en 2018 y un 1,6\% en 2019, excluyendo Venezuela, país en el que las cifras fueron un $1,6 \%$ en 2018 y un $2,1 \%$ en 2019 . Se espera que Sudamérica se contraiga $0,1 \%$ en 2018 y que crezca $1,2 \%$ en 2019 , excluyendo de nuevo a Venezuela, con cifras de $1,2 \%$ de crecimiento en 2018 y 1,9\% de crecimiento en 2019 (tabla 4). 


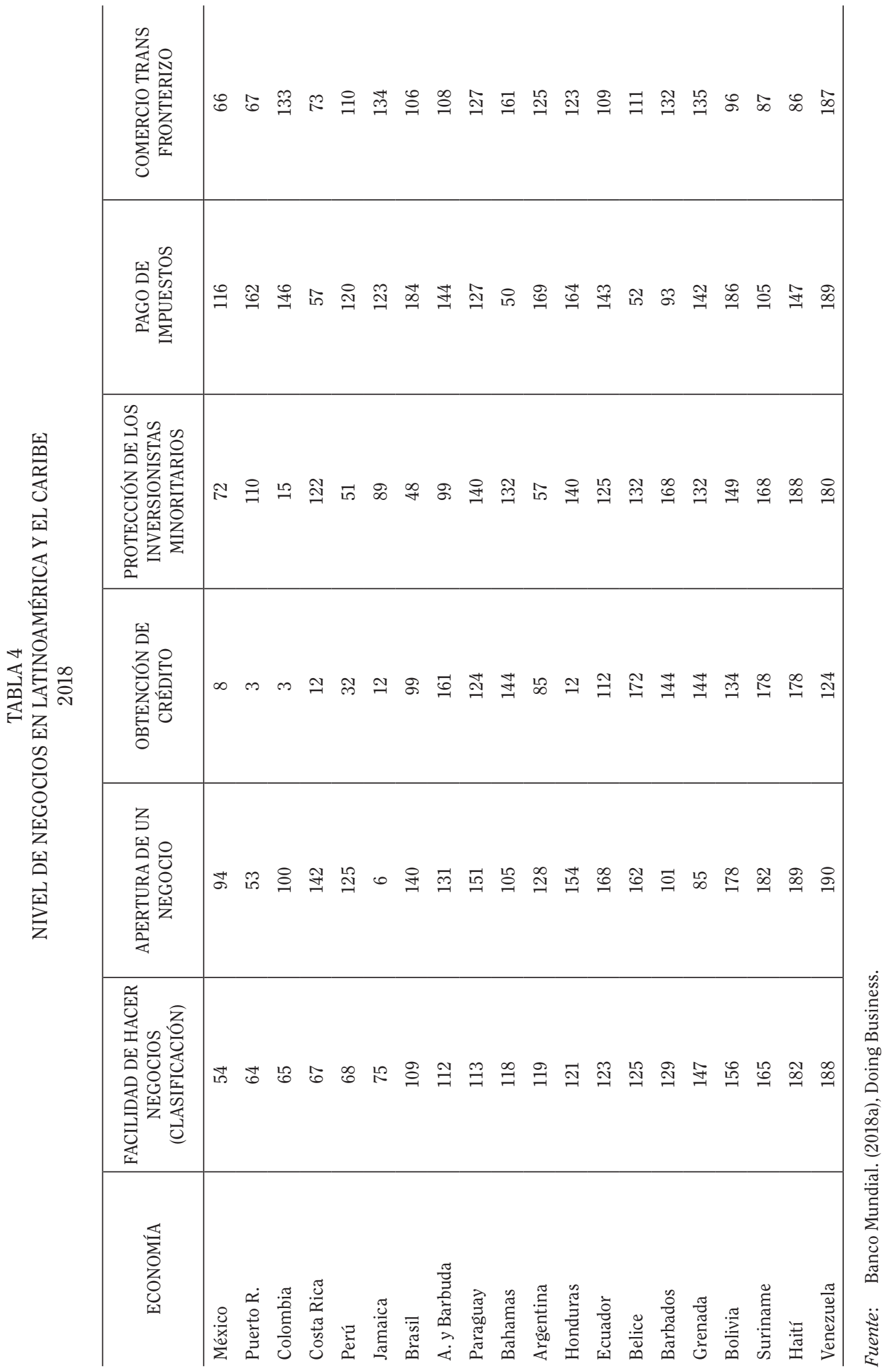


Las razones principales de este incremento más lento en Sudamérica se deben a las perturbaciones del mercado que comenzaron en Argentina en abril, la desaceleración en la expansión de Brasil, el desperfecto continuo de la situación en Venezuela y un entorno externo menos propicio. En cambio, se espera que Centroamérica crezca $2,8 \%$ en 2018 y 3,2\% en 2019; el Caribe, 3,7\% en 2018 y 3,5\% en 2019 y México, $2,3 \%$ en los dos años. Los factores externos que siguen siendo relativamente provechosos para la región son el crecimiento robusto de Estados Unidos, un crecimiento más lento pero aún fuerte de China y una recuperación en los precios de las materias primas (Banco Mundial, 05 de octubre de 2018, párr. 5).

En general, el poco adelanto del comercio local está dificultando la evolución de los países en vías de desarrollo en un mundo global cada vez más competitivo, pero también más agotado por su ambición en relación con la enfebrecida producción que pone en jaque las relaciones comerciales y sus diferentes políticas, entorpeciendo el desarrollo de un mundo económico y social realmente global.

\section{¿QUÉ PASÓ DESPUÉS DE LA CRISIS DEL 2008 EN EL MUNDO GLOBALIZADO?}

La evolución de las sociedades humanas de carácter capitalista se establece a partir del equilibrio entre una eficaz organización social y el desarrollo tecnológico. En el capitalismo global, el mercado no se autorregula existiendo un desequilibrio comercial por culpa del exceso en el crecimiento de la producción en el que la velocidad productiva está acortando los plazos de fabricación en relación con el tiempo de exposición del producto en el mercado y la adaptabilidad del consumidor al producto creado. Este hecho incita a la especulación al perderse el valor (precio) real de un producto, por todo ello, el equilibrio entre la oferta y la demanda debería establecerse a partir de la intervención humana mediante una serie de políticas fiscales $y$ comerciales que deriven hacia un mundo global real basado en el equilibrio social.

Existe en las crisis económicas de 1929 y en la de 2008, la causa coincidente del crecimiento del comercio por volumen y el exceso de producción almacenada. El caso de España muestra que este exceso es el que acaba por destruir en general el tejido empresarial de un país, lo cual provoca el aumento del paro forzoso y, a largo plazo, el debilitamiento de la economía doméstica y el empobrecimiento del propio Estado, el cual agotado y sin ahorros se vuelve caótico e inestable entrando finalmente en recesión económica. Lo anterior sume a la población en una depresión social que descontenta, justifica el alzamiento de partidos políticos de carácter fascista que se creían olvidados, partidos que se alimentan del desorden y el caos que concurre en la sociedad.

Esta desorganización social se refleja en el creciente endeudamiento de empresas, consumidores y Estado, la cual volvió a subir en el 2018 a escala planetaria, situándose en la cifra récord de 247 billones de dólares, unos 211 billones de euros, tres veces más que el PIB mundial. Esta situación deficitaria se complica en los países en vías de desarrollo en el que actualmente existe la dificultad de dar viabilidad a los negocios locales por culpa, entre otras razones, del dominio de las multinacionales de los países desarrollados y la duras condiciones económicas que no permiten una participación de los inversionistas minoritarios, además de la dificultad de obtener créditos bancarios, todo ello a pesar del dato que indica que el $43 \%$ de las exportaciones en el mundo proceden de las naciones más pobres. El Banco Mundial pronostica que en el 2019, según las proyecciones, el crecimiento económico mundial se disminuirá al 2,9\%. El comercio internacional y la inversión en este año se han atenuado ya que las tensiones comerciales siguen siendo altas, $y$ las condiciones de financiamiento se han endurecido.

El crecimiento en las economías de mercados emergentes $y$ en progreso se estancará en un $4,2 \%$ este año, reflejo de una recuperación más débil de lo esperado en los países exportadores de productos básicos y una desaceleración en los países importadores de dichos productos. Los riesgos al desarrollo se han marcado, ya que las coacciones financieras $y$ las tensiones comerciales pueden aumentar, afectando negativamente la 
actividad económica mundial (Banco Mundial, 8 de enero de 2019).

El mundo global podría decaer en parte por la falta de mejores estructuras políticas $y$ económicas que no permiten crecer a la mayoría de los países en vías de desarrollo, lo cual crea una demanda empobrecida que consigue rendir como mano de obra barata pero no como un consumidor rentable que encadena a las poblaciones a una deuda creciente dentro de un contexto social precario. En el informe "Perspectivas sociales y del empleo en el mundo" (Organización Internacional del Trabajo, 2018), se señala que el paro mundial sigue siendo elevado con más de 190 millones de personas desempleadas, aumentando con ello el empleo vulnerable en unos 1400 millones de personas, y esto sucede porque los países desarrollados no admiten equilibrar las fuerzas creando nuevos e igualitarios lazos comerciales, en parte, por su propia crisis de identidad.

\section{REFERENCIAS}

Aranda, J. (2018a). El crac de 1929 en el sistema-mundo: La perspectiva catalana, 1929-1935. El caso de la España industrial. Editorial Siníndice.

Aranda, J. (2018b). La electricidad como causa del exceso de producción (1929-1935). El caso de la España industrial. Técnica industrial, (319), 48-61.

Archivo Nacional de Cataluña. (s.f). Balances de situación, fondo de la España Industrial, 1929-1933, (22.5).

Arrighi, G. y Silver, B.J. (2001). Caos y orden en el sistema-mundo moderno. Editorial Akal.

Banco Mundial. (05 de octubre de 2018). Gestionar riesgos en América Latina y el Caribe será clave en medio de frágil recuperación [comunicado de prensa]. https://www.bancomundial.org/es/news/ press-release/2018/10/05/managing-riskin-latin-america-and-the-caribbean-keyduring-fragile-recovery

Banco Mundial. (2018). Nivel de negocio en el África Subsahariana en 2018. Doing Business 2018. https://www.doingbusiness.org/content/dam/doingBusiness/
media/Annual-Reports/English/DB2018Full-Report.pdf

Banco Mundial. (8 de enero de 2019). Perspectivas poco auspiciosas: la economía mundial se desacelerará al 2,9 $\%$ en 2019 con el debilitamiento del comercio y de la inversión. https:// www.bancomundial.org/es/news/pressrelease/2019/01/08/darkening-prospectsglobal-economy-to-slow-to-29-percentin-2019-as-trade-investment-weaken

Banco Mundial. (2019a). África: Panorama general. https://www.bancomundial.org/ es/region/afr/overview

Banco Mundial. (2019b). Asia Meridional: Panorama general. https://www.bancomundial.org/es/region/sar/overview

Barjot, D. (2005). La aplicación del modelo norteamericano en Europa durante el siglo xx. En L. Delgado y M.D. Elizalde (Coord.), España y estados unidos en el siglo XX (pp.159-161). Consejo Superior de Investigaciones Científicas.

Bolaños, A. (06 de febrero de 2018). La concesión de créditos al consumo vuelve al nivel previo a la crisis. El País. https://elpais.com/economia/2018/02/05/ actualidad/1517855646_489408.html

Carreras, A. y Tafunell, X. (2010). Historia económica de la España Contemporánea (1789-2009). Editorial Crítica.

Estevadeordal, A. (1986). La constitució de societats mercantils a Barcelona, 19121935: anàlisi d'un indicador de formació de capital. [Tesis doctoral]. Universitat de Barcelona, Departament d'Història i Institucions Econòmiques.

Feito, J. L. (2008). Causas y remedios de las crisis económicas: el debate económico Hayek-Keynes, 70 años después, Colección FaEs.

Fernández, D. (12 de mayo de 2018) Compañías en serio peligro de extinción. El País. https:/elpais.com/economia/2018/05/10/ actualidad/1525968594_093223.html

Instituto Nacional de Estadística. (2005-2017). Evolución anual del número de empresas concursadas en España desde 2005 hasta 2017. 
Instituto Nacional de Estadística. (2013-2018). Encuesta Coyuntural sobre Stocks y Existencias, comercio al por mayor en España, 2013-2018.

James, H. (2003). El Fin de la globalización: lecciones de la Gran Depresión. Editoriales Turner y Océano.

La crisis se salda con 45.000 empresas españolas en quiebra (13 de enero de 2016). $A B C$ Economía. https://www.abc.es/economia/ abci-crisis-salda-45000-empresas-espanolas-quiebra.

La crisis del comercio tradicional dispara la quiebra de empresas (02 de agosto de 2018). Economía digital. https://www. economiadigital.es/directivos-yempresas/la-crisis-del-comercio-tradicional-dispara-la-quiebra-de-empresas_570104_102.html

Loayza, N. (2012). Volatilidad y crisis. En P. Martin (Ed.), Pasado y presente: de la gran depresión del siglo $X X$ a la gran recesión del siglo XXI. Fundación BBVA.

Marichal, C. (2010). Nueva historia de las grandes crisis financieras: una perspectiva global, 1873-2008. Editorial Sudamericana-Debate.

Organización Mundial del Comercio [омc]. (2018). Examen estadístico del comercio mundial 2018 (ECM). https://www.wto. org/spanish/res_s/statis_s/wts2018_s/ wts2018_s.pdf

Organización Internacional del Trabajo. (2017). Informe mundial sobre salarios. La desigualdad salarial en el lugar del trabajo, 2016-2017. https://www.ilo.org/ wcmsp5/groups/public/---dgreports/--dcomm/---publ/documents/publication/ wcms_541632.pdf

Organización Internacional del Trabajo. (2018). Perspectivas sociales y del empleo en el mundo. https://www.ilo.org/ wcmsp5/groups/public/---dgreports/--dcomm/---publ/documents/publication/ wcms_631466.pdf

Relaño, V. (01 de setiembre de 2018). España ha aumentado su deuda en 600.000 millones en diez años. Capital Madrid. https:// www.capitalmadrid.com/2018/9/1/50615/ espana-ha-aumentado-su-deuda-en600000-millones-en-diez-anos.html

Ribas, E. (1999). La España Industrial (18511936): E1 Análisis económico-financiero de la compañía. En homenaje al Doctor Nadal: La industrialització $i$ el desenvolupament econòmic d'Espanya, Barcelona, vol 2.

Soler, R. (2012). Estratègies empresarials en la indústria cotonera catalana. El cas de la Fábrica de la Rambla de Vilanova, 1833-1965. Universitat de Barcelona. Departament de Història i Institucions Econòmiques.

Tafunell, X. (2005). Empresa y bolsa. En A. Carreras y X. Tafunell (Coords.), Estadísticas históricas de España (pp. 707-835). Fundación BBva.

Tapia, J.A. y Astarita, R. (2011). La Gran recesión y el capitalismo del siglo XXI: teorías económicas, explicaciones de la crisis y perspectivas de la economía mundial. Catarata y Centro de Investigación para la Paz.

Viaña, D. (17 de febrero de 2018). La crisis triplica la deuda pública: en solo diez años se ha disparado 760.000 millones. El Mundo. https://www.elmundo.es/econo$\mathrm{mia} /$ macroeconomia/2018/02/17/5a870d4 c46163fa01b8b459f.html

Fecha de ingreso: 08/06/2019 Fecha de aprobación: 28/05/2020 